\title{
An approach to building a cross-platform system for the collection and processing of diagnostic information about working technological equipment for industrial enterprises
}

\author{
Petr A. Nikishechkin ${ }^{1,}$, Ilya A. Kovalev ${ }^{1}$, and Anatolii N. Nikich ${ }^{1}$ \\ ${ }^{1}$ MSTU STANKIN, 127055 Vadkovskiy per. 3a, Moscow, Russia
}

\begin{abstract}
The article is devoted to the trends of building a modern smart manufacturing and solving information transmission problems from technological equipment to the higher levels of enterprise management. The theoretical and practical aspects of developing solutions for collecting the diagnostic information about the work of technological equipment, the construction of its computational kernel, and results of its tests are presented.
\end{abstract}

Data acquisition from the working area where particular technical operations are carried out is a major and important problem for a modern high-tech manufacturing $[1,2]$. Through the use of obtained data not only different diagnostic data can be traced (errors and warnings of CNC systems, temperature, vibration while cutting work, tool wear and etc.), but also the information about machine capacity and work of its controller (for example machine hours). Modern data transfer and information processing about work of the production equipment to higher levels of an enterprise is on a direct basis of economical indicators not only of a single section, workshop, but also of the whole enterprise, as it allows to react immediately to emergency situations and to increase productivity by process flow optimization [3-5].

Modern technologies represent clusters for various processing operations. Often one controller can maintain up to 5 machines where it is necessary to monitor technology process. Herewith there can be more than 20 controllers on the line, operated process units - more than 100 (at large machine-building enterprises «Uralvagonzavod», «KEMZ», «AvtoVAZ» - more than 1000).

Currently flourishes the tendency to enterprise development in accordance with the conception «Industry 4.0» and construction of digital production with high degree correlation between all levels of the enterprise. Large complex of production machinery from various manufacturers with different data exchange protocols often complicates operating monitoring processes and entangles the process of technical data transmission to higher levels of the enterprise (figure 1).

The paper suggests to work out the system the main task of which is classified accumulation and heterogeneous information processing into the single data-base [6] and its transmission to the higher enterprise levels.

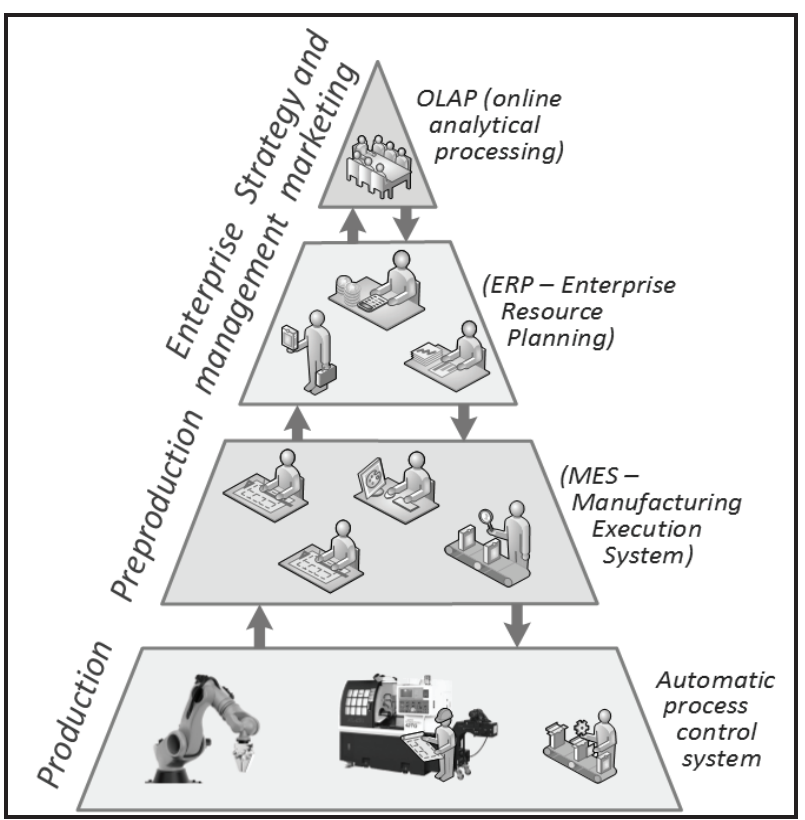

Fig. 1. Levels of modern digital production arrangement.

In the furtherance of this goal it is suggested to develop cross-platform software-based controller able to carry out compilation of data from different processing equipment. The conducted analysis showed that there are no analogues of the developed product in the domestic market. The concurrence with foreign solutions is avoided because of the another price tier and also due to the fact that most of the foreign workings represent proprietary software. The general structure of proposed solution is showed in figure 2 and can be both an autonomous solution that transmits various diagnostic data to the net and integrated to the control system of the higher level, for example, CNC system.

\footnotetext{
* Corresponding author: pnikishechkin@gmail.com
} 


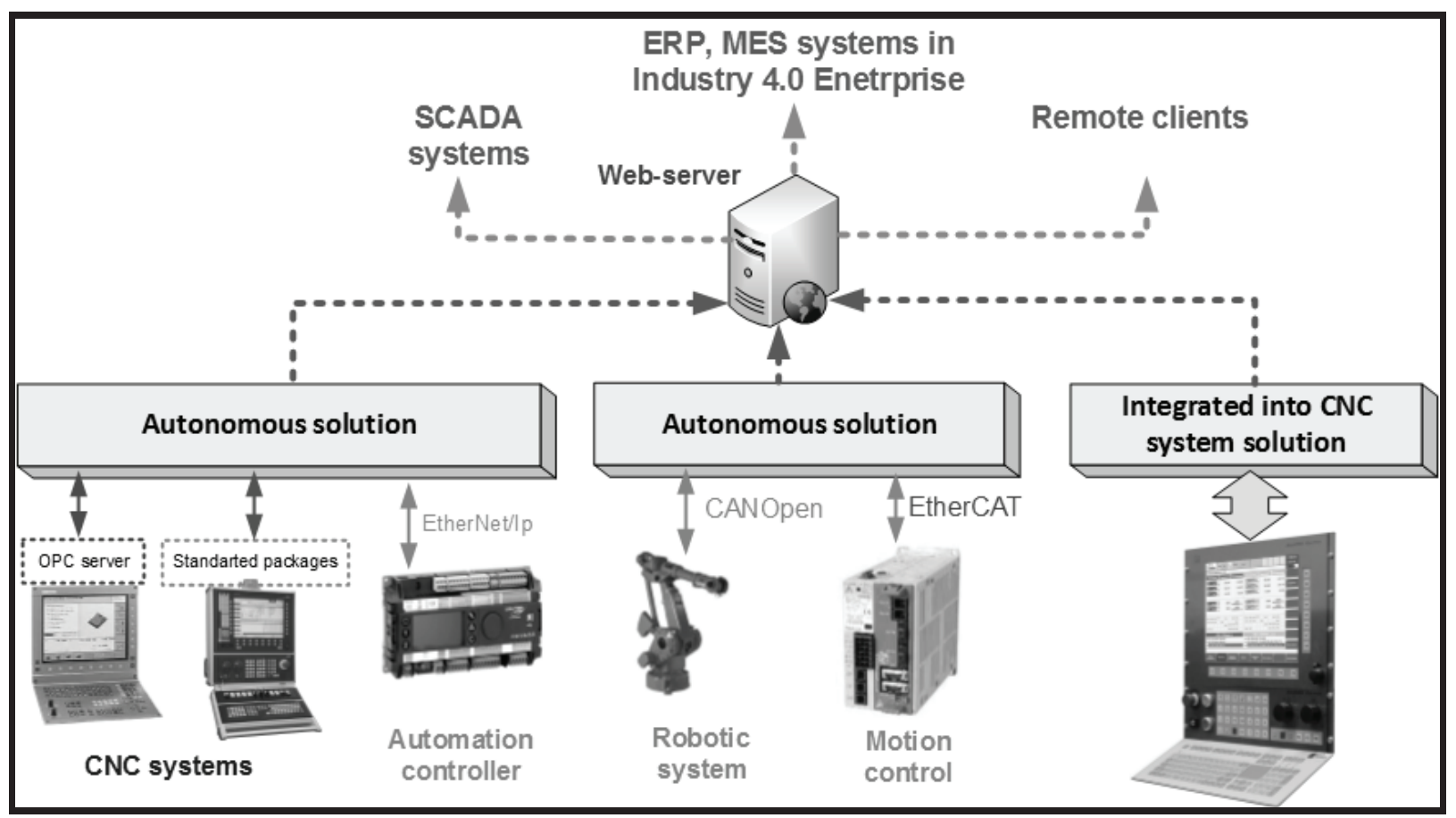

Fig. 2. Levels of modern digital production arrangement.

The main implementation of the suggested solution is at the application layer and it is a programmed computation module that allows to connect to the equipment of various types using best known interconnect protocols (EtherCAT, SERCOS, CANBus) and to aggregate the data to the integrated Webserver and then the information will be transmitted to the higher production levels (SCADA, MES, remote client users).

The main implementation of the suggested solution is at the application layer and it is a programmed computation module that allows to connect to the equipment of various types using best known interconnect protocols (EtherCAT, SERCOS, CANBus) and to aggregate the data to the integrated Webserver and then the information will be transmitted to the higher production levels (SCADA, MES, remote client users).

Developed the organization of the main modules of the suggested solution. The central part shows the main computation module implementation - computational kernel that works in real-time mode and carries out data acquisition from the equipment of various types. This module interacts with terminal clients and also with automated equipment.

The main task of the basic computational module (kernel) is data acquisition and information processing in real-time mode from the equipment of various types through different fieldbuses. Flexibility, generality of the solution and the possibility of using it with a set of control equipment from different manufacturers is possible due to the abstract level implementation (execution units, interconnect protocols and etc.), that is often observed in domestic enterprises, even within the same workshop.

It is suggested to implement the basic computational module in accordance with the modular approach: a terminal module of the top level of the user space, a mathematical module where basic calculations occur, a cross-platform module, a driver module that operates in the address space of the selected OS. These modules are implemented in $\mathrm{C}++$ using object-oriented programming methods. Currently, the solution is being adapted to the work with NCT201 CNC systems (NCT, Hungary), AxiOMAControl (MSTU "Stankin", Russia), as well as with servocontrollers IntDrive (BoschRexroth) and DS (NCT, Hungary) and obtaining diagnostic data from them.

In addition to working with standard equipment to get the diagnostic information on the operation of the technological unit it is supposed a possibility of different sensor types connection (vibration, thermo), the interaction with them can be carried out with remote input/output devices (using analog inputs). Thus, it is possible to expand the area of the diagnosed equipment and to receive more extensive and reliable information about the operation of technological units and, in general, of the workshop/enterprise.

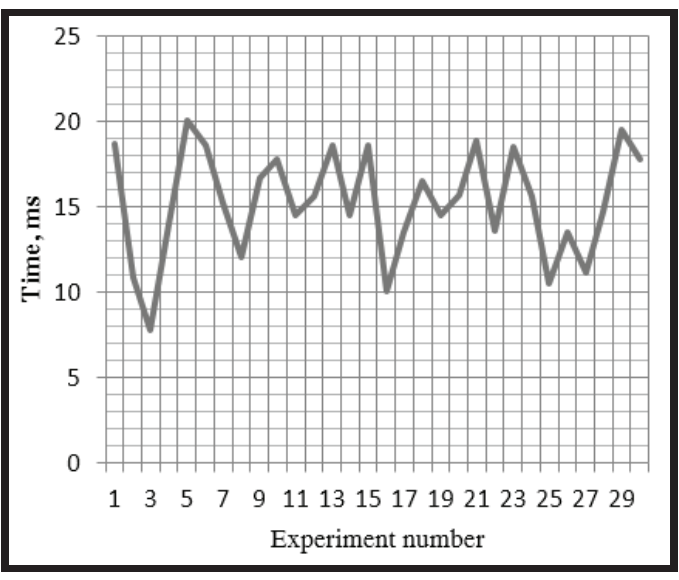

Fig. 3. Scanning tests of the net device elements 
During tests it was found out that the amount of used program elements tied to the structure of processing facilities can increase sufficiently (the implementation of connection of five milling machines worked out in about 2000 components in the integrated environment). Scanning such amount of elements (figure 3 there are 100 elements, figure $4-1500$ elements) took up about 2 seconds.

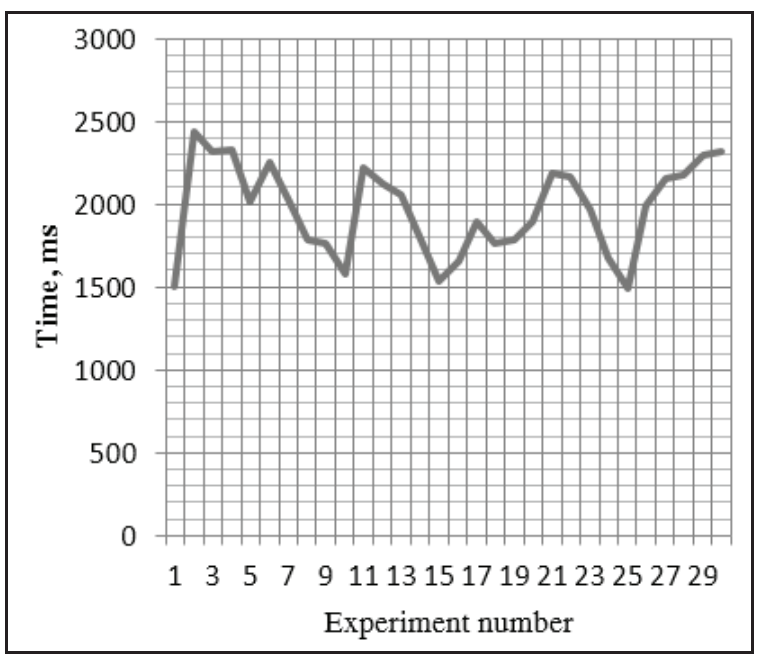

Fig. 4. Scanning tests of the net device elements

Taking into consideration the fact that there can be more than 15 production units in one data line, the total number of polled processing facilities projected to the control program can amount to more than 10 thousand.

It was decided to use the method of asynchronous messages with maximum data burst filling instead of the method of polling type request (one request-one answer) to increase the high speed response. In case of the new approach for introduced example the average time of getting data from all nodes fall by $50 \mathrm{~ms}$, also to get more process pieces this time can be maintained within $100 \mathrm{~ms}$, by means of asynchronous messages only from those devices that changed its state [13].

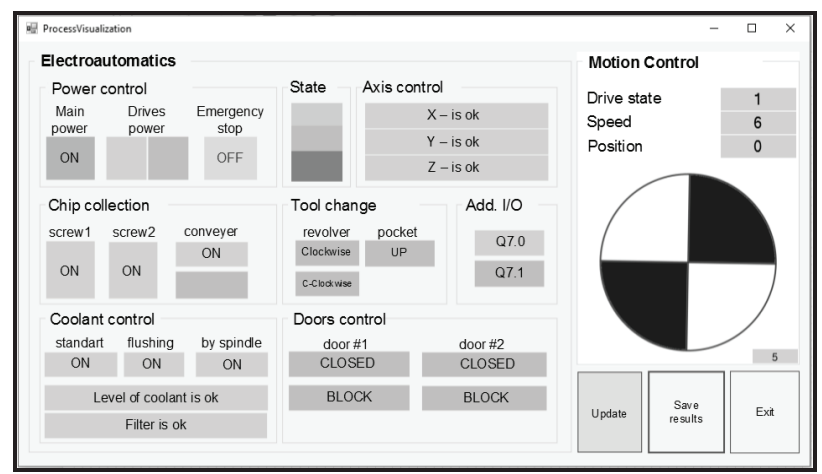

Fig. 5 Example of window process visualisation

The figure 5 shows the implementation of process visualization for a milling machine. The proposed approach allows to display what is happening in the subsystem of electroautomatics, working on fieldbus EtherCAT, as well as take readings from engines working on the Sercos fieldbus
Along with that one of the important characteristic while computational kernel development of the suggested solution is its cross-platform both at the system and hardware level. It allows to use the various base of its building-up and integrate it to different working systems. At the system level the work fulfillment in various operation systems is realized due to the use of cross-platform pools, mutexes, timers and function wrapping and devices of shared memory: Windows-family, OS based on Linux, also with Windows system extension - system RTX of IntervalZero company [14-15].

Cross-platform at the system level also allows to perform module porting to different hardware platforms - it is realized the possibility of the main module work on the processors of various type: x86 (Intel, AMD) or ARM including domestic ones (Elbrus 4C, Baykal-T, M). It helps to use module both on personal computers and on single-board computers, and to draw out single compact devices for automation of technological processes with the possibility of remote control and access to data that is an important condition in smart manufacturing.

Consequently, in case of this approach the following tasks can be solved: heterogeneous hardware unification into the consolidated information area, monitoring of the equipment operation status, data acquisition about machine and operator capacity, rapid response to emergency situations, forecasting of possible contingency, the possibility of transferring information about production to the upper levels of the enterprise (matching for development concept Industry 4.0), and also downtown time minimization [12,14-16].

Practical implementation and use of the suggested solution at production enterprises will provide an opportunity to get a new scheme for data acquisition and information processing from the machine line and other manufacturing equipment with the use of domestic hardware and software components. Implementing of cross-platform approach in the solution will make it possible to decrease, and in the future to avoid dependence on foreign elements, and thus to provide technological and information security of domestic enterprises.

\section{References}

1. G.M. Martinov., L.I. Martinova, Development of the base computing platform of CNC for construction of specialized control systems, Vestnik MSTU "Stankin", №1 (24), P. 92-97. (2014)

2. G.M. Martinov, A.B. Lyubimov, A.I. Bondarenko, A.E. Sorokoumov, I.A. Kovalev, An approach to building a multiprotocol CNC system, Automation and Remote Control, Vol.76, No. 1, P. 172-178 (2015)

3. G.M. Martinov, A.S. Grigoryev, P.A. Nikishechkin. Real-time diagnosis and forecasting algorithms of the tool wear in the CNC systems. Advances in Swarm and Computational Intelligence. Vol.9142. P. 115-126 (2015) 
4. S.N. Grigoriev., G.M. Martinov, An ARM-based Multi-channel CNC Solution for Multi-tasking Turning and Milling Machines, Procedia CIRP. Vol.46, P. 525-528 (2016)

5. S.N. Grigoriev., G.M. Martinov, The Control Platform for Decomposition and Synthesis of Specialized CNC Systems, Procedia CIRP, Vol.41, P. 858-863 (2016)

6. S.N. Grigorev, V.A. Dolgov, A.V. Krasnov, A.A. Kabanov, N.S. Andreev, A Method of Technologic Audit of Technical Re-Equipment Projects in Aircraft Production Enterprises, Izv.Vuz. Av. Tekhnika, Vol.58, no. 2, P. 103-108 (2015)

7. G.M. Martinov, A.I. Obuhov, L.I. Martinova, A.S. Grigoriev, An Approach to Building a Specialized CNC System for Laser Engraving Machining. Procedia CIRP, Vol. 41, P. 998-1003 (2016)

8. G.M. Martinov, R.A. Nezhmetdinov, S.V. Sokolov, The Principles of Constructing a Toolchain for Monitoring and Setup Mechatronic Equipment Parameters Based on Integration of Specialized Software Components into Control System Structure, Mekhatronika Avtomatizatsiya, Upravlenie, No. 7, P. 45-50 (2012)

9. L.I. Martinova, S.V. Sokolov, P.A. Nikishechkin, Tools for Monitoring and Parameter Visualization in Computer Control Systems of Industrial Robots, Advances in Swarm and Computational Intelligence. 6th International Conference, Part II, P.200-207. (2015)

10. G.M. Martinov, R.A. Nezhmetdinov, P.A. Nikishechkin, Development of visualization means and debugging of control programs for magnetics integrated into CNC system, Vestnik MSTU "Stankin", №4 (23), P. 134-138 (2012)

11. G.M. Martinov, P.A. Nikishechkin, A.S. Grigoryev, N.Y. Chervonnova, Organization of interaction of the main components of the CNC AxiOMA Control for integration into its new technologies and solutions, Automation in the industry, №5, P.10-15 (2015)

12. R.A. Nezhmetdinov, P.A. Nikishechkin, R.L. Pushkov, S.V. Evstafieva, Practical aspects of the software-based controller to control electroautomatics vertical milling machines Quaser MV184, Automation in the industry, №5, P. 15-18. (2016)

13. N.V. Kozak, R.A. Abdullaev, I.A. Kovalev, N.Yu. Chervonnova, Realization of the logical task of CNC and task of industrial safety on the basis of external computing SoftPLC modules, Automation in the industry, №5, P. 28-30 (2016)

14. G.M. Martinov, N.V. Kozak, Construction of a specialized CNC system for a five-axis planermilling machining center, STIN, №8, P.2-6 (2015)

15. I.A Kovalev, S.V. Sokolov, L.I. Martinova, S.V. Rybnikov, Construction of a specialized CNC system for thread grinding machines, Automation in the industry, №5, P.38-40 (2015)
16. G.M. Martinov, N.V. Kozak, R.A. Abdullaev, I.A. Kovalev, Construction of a specialized distributed control system for a precision machining center $V M G$ 50, Automation in the industry, №6, P. 16-20 (2014) 